\title{
2 DIMENSIONAL DISLOCATION DYNAMICS - A NEW TECHNIQUE FOR THE SIMULATION OF DEFORMATION MICROTEXTURES
}

\author{
F. ROTERS and D. RAABE \\ Collaborative Research Center for Materials Modeling \\ Institut für Metallkunde und Metallphysik \\ Rheinisch-Westfälische Technische Hochschule Aachen, Kopernikusstr. 14 \\ 52056 Aachen, Germany
}

(Received 18 April 1996)

\begin{abstract}
A new technique for the simulation of microtexture evolution during cold deformation which is based on 2 dimensional (2D) dislocation dynamics is presented. In the simulation all involved dislocations are regarded as infinite straight line detects which are embedded in an otherwise isotropic linear elastic medium. As the model is $2 \mathrm{D}$ only edge dislocations are considered.

In the first simulation step the net local stresses are derived and used to calculate the resulting dislocation motion. Dislocation multiplication, annihilation and reactions are taken into account. Thermal activation is included. In the second step the local misorientations arising from the dislocation distribution are calculated.

This method shows in microscopic detail how misorientations are generated and distributed within grains during plastic deformation.
\end{abstract}

KEY WORDS: Deformation microtexture, simulation, 2D dislocation dynamics, edge dislocations.

\section{INTRODUCTION}

Classical texture investigations which are based on the determination of the orientation distribution function (ODF) from experimental X-ray or neutron pole figures provide quantitative, statistical data (Bunge 1965, 1982) Nowadays there is a trend towards more and more localized texture measurements. The introduction and optimization of local orientation determination in the scanning electron microscope (SEM) and transmission electron microscope (TEM) has stimulated the extension of texture research both to the mesoscopic (SEM) and microscopic (TEM) regime. In the SEM grain orientations can be determined by analyzing electron back scatter diffraction patterns (Venables and Harland, 1973. Dingley 1984, Dingley and Randle 1992. Engler and Gottstein 1992, and Hjelen, Orsund and Nes 1991). In the TEM local orientations are determined by analysis of microbeam diffraction Kikuchi patterns or determination of selected area diffraction pole figures (Humphreys 1984, Schwarzer 1991).

Whilst numerous of the above mentioned experimental SEM and TEM techniques have been substantially improved in the last decade, there exists still a lack of corresponding simulation techniques which allow to numerically investigate the evolution of deformation microtextures. 
Recently, some authors have addressed the simulation of the evolution of local texture changes during cold deformation e.g. the generation of deformation bands (Lee, Duggan and Smallman 1993) or orientation distributions within single grains (Rosen et al. 1995, Raabe 1994) or small groups of grains (Beaudoin, Mecking and Kocks 1995). Other researchers have focused on the simulation of local discontinuous orientation changes during primary static recrystallization (Juul Jensen 1992, Marx, Raabe and Gottstein 1995).

However, most other approaches which so far aimed to simulate the evolution of texture during rolling (Taylor 1938) and annealing (Köhler and Bunge 1994) were based on statistical rather than local models.

In this paper we present a simulation method which allows to determine microtextures on the basis of the simulation of dislocation dynamics. This technique represents a reasonable new microscopical approach to the prediction of microtexture evolution within single grains, since it are the dislocations which are the elementary carriers of local misorientations (Nye 1953, Ashby 1970). Due to the restrictions of computer power the method is at first employed in two dimensions (2D), since only the limitation to 2D information allows to obtain results for larger degrees of deformation within reasonable computing times. Corresponding 3D simulations are much more complicated and time consuming and hence provide microtexture data only for strains below $2 \%$ (Raabe 1996). The simulation of dislocation dynamics supplies information about the evolution of the dislocation distribution with maximum spatial resolution. From this distribution both the local stresses and the local misorientations can be easily calculated within each time increment.

In the following chapters we outline the basic concept of the 2D simulation technique. Subsequently, we will discuss some preliminary results. Finally, we will critically review the merits and shortcomings of the present approach and its potential for further application.

\section{SIMULATION}

\section{Basic Approach}

In the present numerical simulation we calculate the microtexture as imposed by dislocations with microscopic resolution within single grains. The involved dislocations are treated as infinite linear defects which are embedded in an otherwise isotropic linear elastic medium. Using isolated dislocations for the calculation of deformation microtexture represents the most detailed approach because dislocations are the elementary carriers of both deformation and misorientation. The simulation of the evolution of the deformation microtexture can be separated into two successive steps. The first step comprises the simulation of the evolution of the dislocation distribution and the second step covers the calculation of the misorientation distribution as determined by the spatial arrangement of the dislocations. Both steps are realized by independent programs and are described in the following chapters.

\section{The Evolution of the Dislocation Distribution}

At the beginning of the simulation the initial dislocation distribution is generated. As the simulation is 2D, only edge dislocations are taken into account. This simplification is in accord with experimental (Zehetbauer and Seumer 1993, Zehetbauer 1993) and theoretical (Mughrabi 1983 and Zehetbauer 1993) findings. As screw dislocations reveal 
a large annihilation distance (Essmann and Mughrabi 1979), a centralforce type interaction and a high mobility which altogether makes it easy for them to depart from their actual slip plane, their contribution to dislocation structure formation is negligible at least in stages I, II and III. According to the chosen initial dislocation density, $\rho$, and number of involved glide systems the dislocations are randomly distributed within the rectangular simulation cell. The simulation cell represents one single grain with geometric boundary conditions. Even though plastic deformation processes are simulated, in a simplified approach, the cell maintains its geometric shape, size and orientation during the entire simulation. The influence of neighboring grains is not taken into account apart from maintaining the simulation cell geometry as mentioned before.

The number of dislocations with positive and negative Burgers vector is balanced inside the simulation cell. As new dislocations are always introduced in pairs, which indicates the activation of Frank-Read sources, this condition is valid during the entire simulation. In each simulation step all dislocations are moved due to the local value of the stress field, $\underline{\sigma}_{t o t}(\underline{r})$, at their respective positions $\underline{r}$. The local value of the stress field represents a superposition of several internal and external stress contributions. Quantitatively, the most important internal stress contribution arises from the other dislocations within the system, $\underline{\sigma}_{d i s}(\underline{r})$. The contribution of a single dislocation, $\underline{\underline{\sigma}}_{i}(\tilde{r})$, is calculated according to the framework given for straight dislocations of infinite length in an isotropic linear elastic medium (Hirth and Lothe, 1968):

with

$$
\underline{\tilde{\sigma}}_{i}(\tilde{\tau})=\left(\begin{array}{ll}
\tilde{\sigma}_{x x} & \tilde{\sigma}_{x y} \\
\tilde{\sigma}_{y x} & \tilde{\sigma}_{y y}
\end{array}\right) \quad, \underline{\tilde{\tau}}=\left(\begin{array}{l}
\Delta \tilde{x} \\
\Delta \tilde{y}
\end{array}\right)
$$

$$
\begin{aligned}
& \tilde{\sigma}_{x x}=-\frac{G}{2 \pi(1-v)} \cdot \frac{\Delta \tilde{y}\left(3 \Delta \tilde{x}^{2}+\Delta \tilde{y}^{2}\right) \tilde{b}}{\left(\Delta \tilde{x}^{2}+\Delta \tilde{y}^{2}\right)^{2}} \\
& \tilde{\sigma}_{y y}=\frac{G}{2 \pi(1-v)} \cdot \frac{\Delta \tilde{y}\left(\Delta \tilde{x}^{2}-\Delta \tilde{y}^{2}\right) \tilde{b}}{\left(\Delta \tilde{x}^{2}+\Delta \tilde{y}^{2}\right)^{2}} \\
& \tilde{\sigma}_{x y}=\tilde{\sigma}_{y x}=\frac{G}{2 \pi(1-v)} \cdot \frac{\Delta \tilde{x}\left(\Delta \tilde{x}^{2}-\Delta \tilde{y}^{2}\right) \tilde{b}}{\left(\Delta \tilde{x}^{2}+\Delta \tilde{y}^{2}\right)^{2}}
\end{aligned}
$$

whene $G$ is the shear modulus, $b$ the magnitude of the Burgers vector and $v$ Poisson's ratio. Variables which are indicated by a ' $\sim$ ' are given in the coordinate system of the dislocation. For expressing, the stress tensor in the crystal reference coordinate system a rotation matrix, $\underline{D}_{k}$, is defined for each slip system $k$ :

$$
\underline{D}_{k}=\left(\begin{array}{cc}
\cos \alpha_{k} & \sin \alpha_{k} \\
-\sin \alpha_{k} & \cos \alpha_{k}
\end{array}\right)
$$

where $\alpha_{k}$ is the inclination angle of the respective glide plane with respect to the crystal reference coordinate system. The contribution of the dislocations to the internal stress, $\underline{\sigma}_{d i s}(\underline{r})$, can then be calculated according to:

$$
\underline{\sigma}_{d i s}(\underline{r})=\sum_{k} \underline{\underline{D}}_{k}^{-1}\left(\sum_{i_{k}} \underline{\underline{\sigma}}_{i}(\underline{\tilde{r}})\right) \underline{D}_{k}
$$


Additional contributions to the total local stress field, $\underline{\sigma}_{t o t}(\underline{r})$, stem from a) the large angle grain boundaries (Nabarro 1952):

with

$$
\sigma_{G B}=2 \mathrm{G} \psi \eta \exp (-\eta)
$$

$$
\eta=2 \pi|x| / b
$$

where $\psi$ is the misorientation angle between neighboring grains and $x$ the distance from the grain boundary and b) the external stress field, $\underline{\underline{\sigma}}_{e x t}$, so that:

$$
\underline{\sigma}_{t o t}(\underline{r})=\underline{\sigma}_{d i s}(\underline{r})+\underline{\sigma}_{G B}+\underline{\underline{\sigma}}_{e x t}
$$

The Peierls stress is neglected in the above equation, which is a good estimate for higher temperatures. Now the resulting force on the dislocation can be calculated by employing the Peach-Koehler-equation, $\underline{F}=(\underline{\sigma} \cdot \underline{b}) \times \underline{s}$, with $\underline{s}$ being the line element of the dislocation. $\underline{s}=(001)$ for all dislocations. If $\underline{F}$ is transformed back to the dislocation coordinate system, the components of the force vector parallel to the direction of glide, $\widetilde{F}_{\text {glide, }}$, and climb, $\widetilde{F}_{\text {climb }}$, can be obtained.

Dislocation glide is modeled as a thermally activated process. Therefore the dislocation velocity in the direction of glide, $\widetilde{v}_{\text {glide }}$, can be expressed in the following manner:

$$
\tilde{v}_{\text {glide }}=v_{0} \cdot \exp -\frac{Q-\sigma V}{k T}=v_{0} \cdot \exp -\frac{Q-\tilde{F}_{\text {glide }} A}{k T}
$$

where $Q$ is the activation energy and $\sigma V=\widetilde{F}_{\text {glide }} A$ the mechanical work which results from the net local stress, $\underline{\sigma}_{t o t}(\underline{r})$. For both the activation area $A$ and the pre-exponential factor, $v_{0}$, it is stipulated that they are proportional to the dislocation spacing.

In opposition to this glide law the non-conservative climb process is controlled by diffusion. The velocity of dislocation climb is thus directly proportional to the respective force component with the diffusion coefficient $D$ as proportional constant, viz.:

$$
\tilde{\nabla}_{c l i m b}=B \widetilde{F}_{c l i m b}=\frac{D}{k T} \widetilde{F}_{c l i m b}=\frac{\tilde{F}_{c l i m b}}{k T} D_{0} \exp \left(-\frac{H}{k T}\right)
$$

Where $H$ is the activation enthalpy for vacancy formation and migration.

Once the dislocation velocities are determined in accord with the respective local stress fields it has to be controlled that all resulting dislocation reactions are considered by the simulation. Therefore the time increment of each simulation step is dynamically chosen. To do so the minimum distance between two dislocations is determined. The distance traveled by the fastest dislocation is then limited to half this value. After the time increment is determined, all dislocations are moved according to their individual velocity.

With all dislocations in their new positions, dislocation reactions are carried out. There are two types of reactions explicitly incorporated in the simulation. The first one is dislocation annihilation. If two dislocations on identical glide systems but with opposite Burgers vectors approach closer than the minimum allowed spacing $\left(d_{\text {annihil }} \approx 10 \cdot b\right.$, Essmann and Mughrabi 1979) they annihilate and are therefore removed from the simulation. Secondly the formation of locks is considered. Physically, lock formation 
takes place, when two dislocation on different glide systems react to form a new dislocation with a resulting Burgers vector, which is no longer a translation vector of an activated slip system. In the present study this process is realized by the immobilization of dislocations on different glide systems when they approach too close to each other $\left(d_{\text {lock }} \approx 5 \cdot b\right)$.

2D simulations of dislocation dynamics do not naturally include dislocation multiplication. This shortcoming is due to the fact that all line elements are parallel. That means new dislocations have to be introduced artificially. This is done in a way that assures that for every simulation step the Taylor formula, $\sigma=\alpha G b \sqrt{\rho}$, is fulfilled. The new dislocations are distributed to the active glide systems according to their respective contribution to the total shear. Subsequently, the next simulation step starts.

\section{The Microtexture}

In the second step of the simulation, the microtexture i.e. the local orientation distribution, is calculated from the dislocation distribution obtained from the preceding dislocation dynamics simulation. For deriving the microtexture from a given dislocation distribution the entire simulation cell, i.e. the grain investigated, is divided into small areas. A simple example is given in figure 1, where the grain is divided into elongated cells of equal size parallel to the $y$-axis. This arrangement allows to investigate the misorientations along the $x$-axis. In order to determine the misorientation increment the $x$-components of the Burgers vectors, $b_{x_{i}}$, of all $i$ dislocations inside each cell are summed up. The induced misorientation increment, $\delta_{x}$, inside the cell indexed by $x$ is then calculated as that of an ideal small angle boundary with the same resulting net Burgers vector, e.g. according to:

$$
\delta_{x}=\frac{\sum_{i} b_{x_{i}}}{l_{y}}
$$

where $l_{y}$ is the height of the grain parallel to the $y$-direction (Figure 1). The total misorientation, $\Delta_{x}$, at any position which is indicated by $X$ can then be calculated according to:

$$
\Delta_{x}=\sum_{x \leq x} \delta_{x}
$$

The misorientations, $\Delta_{Y}$, along the y-axis can be derived analogously.

\section{RESULTS}

The present microtexture results are obtained from the simulation of a deformation experiment with a subsequent annealing treatment using the material constants of pure Aluminum. Due to the early state of this microscopic simulation approach only weak plastic strains were considered in the computer experiment. The initial value for the external stress component, $\sigma_{x y}$, was $10 \mathrm{MPa}$ which was then raised by a rate of as much as $10 \mathrm{GPa} / \mathrm{s}$ for $5.5 \mathrm{~ms}$, thus ending up with $\sigma_{x y}=65 \mathrm{MPa}$. The deformation temperature amounted to $500 \mathrm{~K}\left(T / T_{m}=0.54\right)$ and the final plastic deformation, $\varepsilon_{x y}$, to only $0.3 \%$. After the deformation the external stress was dropped to zero $\left(\sigma_{x y}=\right.$ $0 \mathrm{MPa})$ and the temperature was raised to $T=700 \mathrm{~K}\left(T / T_{m}=0.75\right)$ for the duration of $1 \mathrm{~s}$. This change of the simulation parameters corresponds to a recovery experiment. 


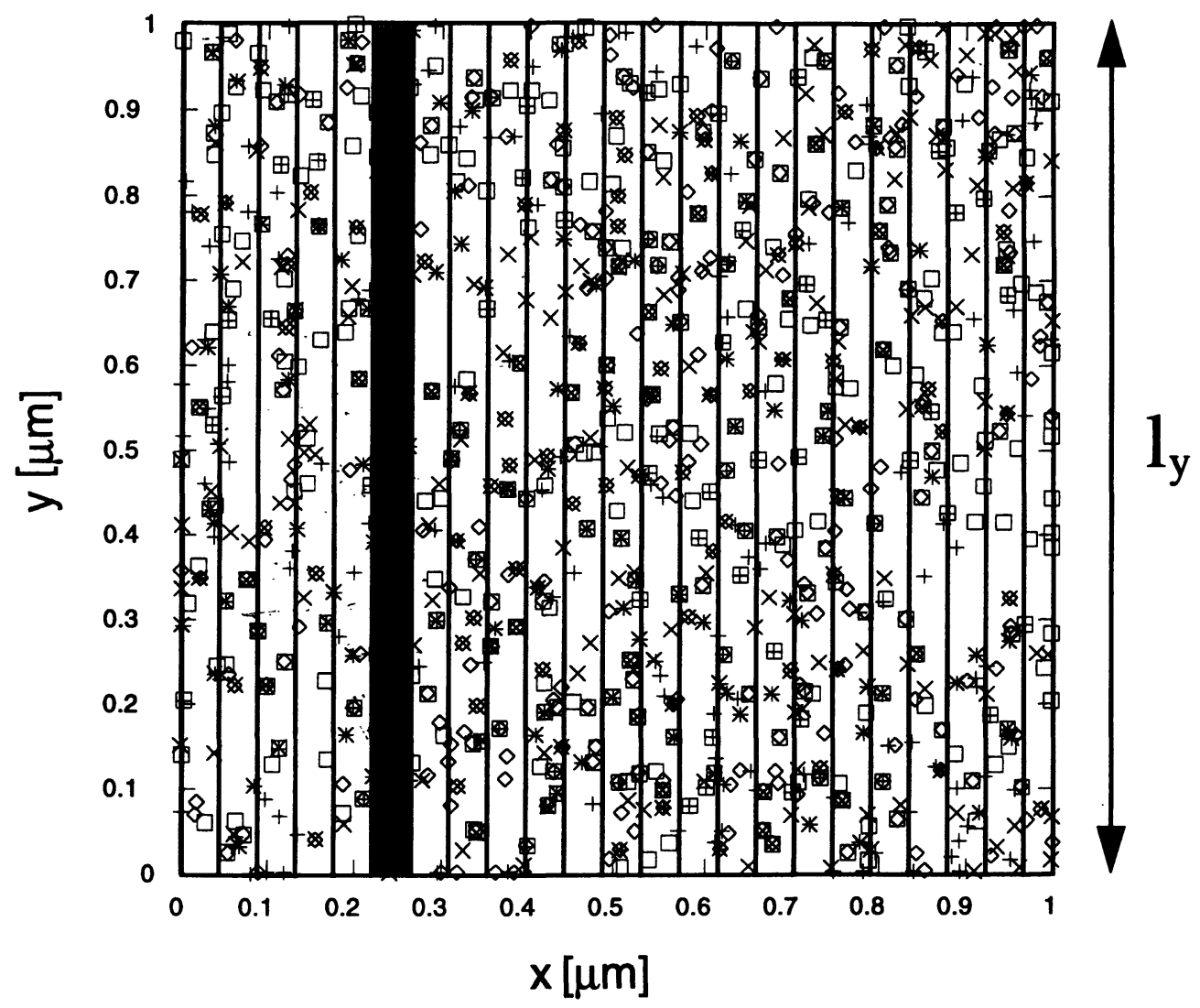

Figure 1 Example for the division of the simulation cell into small regions. Here elongated rectangulars parallel to the $y$-axis are chosen.

This annealing treatment resulted in a decrease of $\varepsilon_{x y}$ by about $0.1 \%$. Figures 2 to 4 show the dislocation structure and the corresponding misorientation distributions at the beginning of the simulation (Figure 2), after the deformation (Figure 3), and after the annealing treatment (Figure 4).

Figure 2a shows the initial random distribution of the dislocations prior to the deformation. The initial dislocation density amounted to $\rho=10^{13} \mathrm{~m}^{-2}$. Figure $2 \mathrm{~b}$ and 2c show, as one would expect for a random distribution, that there were almost no misorientations within the grain $\left(\Delta_{x}<0.04^{\circ}, \Delta_{y}<0.03^{\circ}\right)$. Figure 3 shows the situation after the deformation simulation. The dislocation density was substantially increased $\left(\rho=3 \cdot 10^{14} \mathrm{~m}^{-2}\right.$, Figure $\left.3 \mathrm{a}\right)$ and as can be easily seen from Figures $3 b$ and $3 c$ considerable local misorientations have been build up during the deformation. The still rather low maximum value of the misorientation distribution of about $0.43^{\circ}$ can be understood it one keeps in mind that the plastic deformation only amounted to $0.3 \%$. 
DISLOCATION DYNAMICS
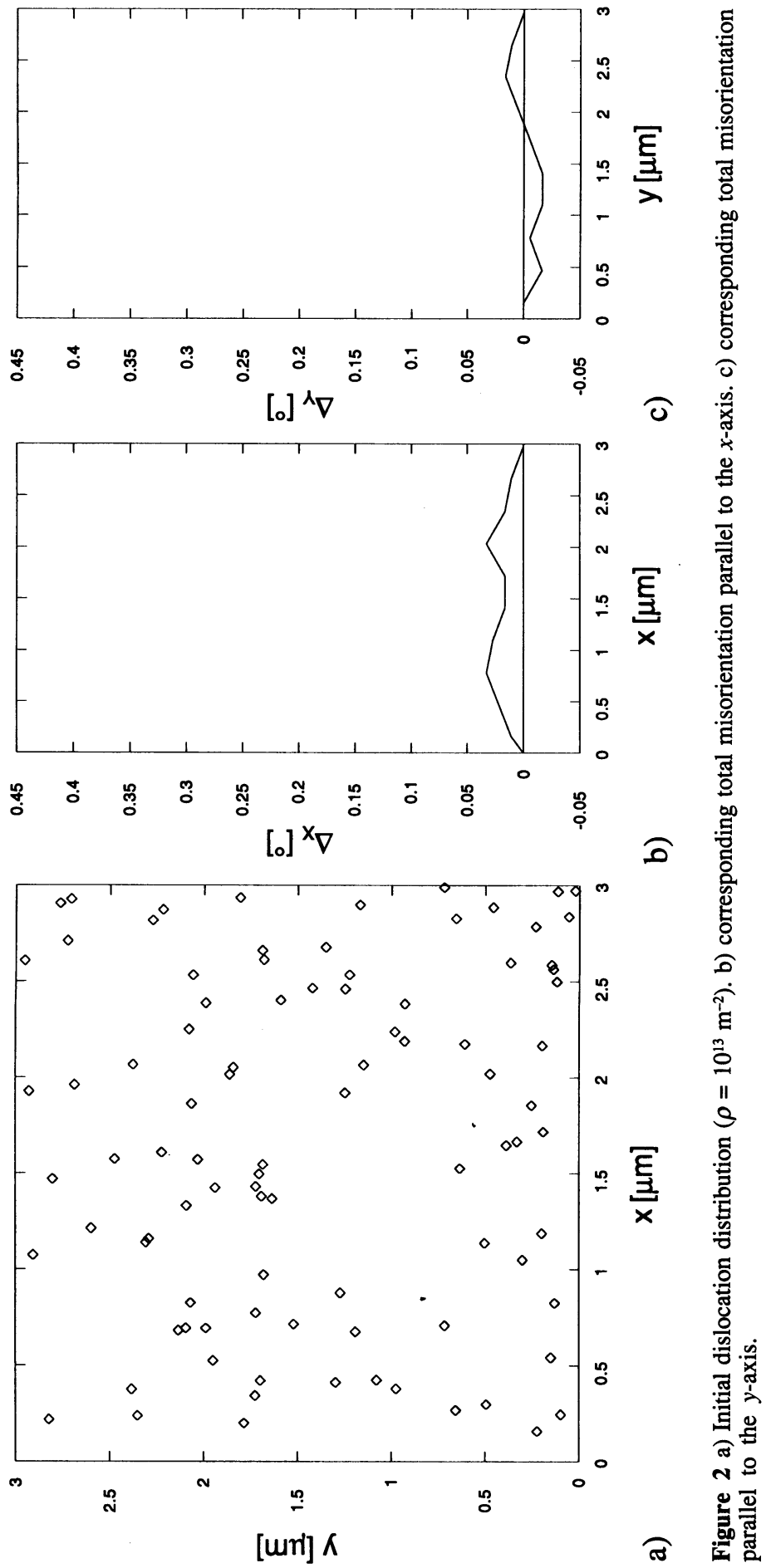

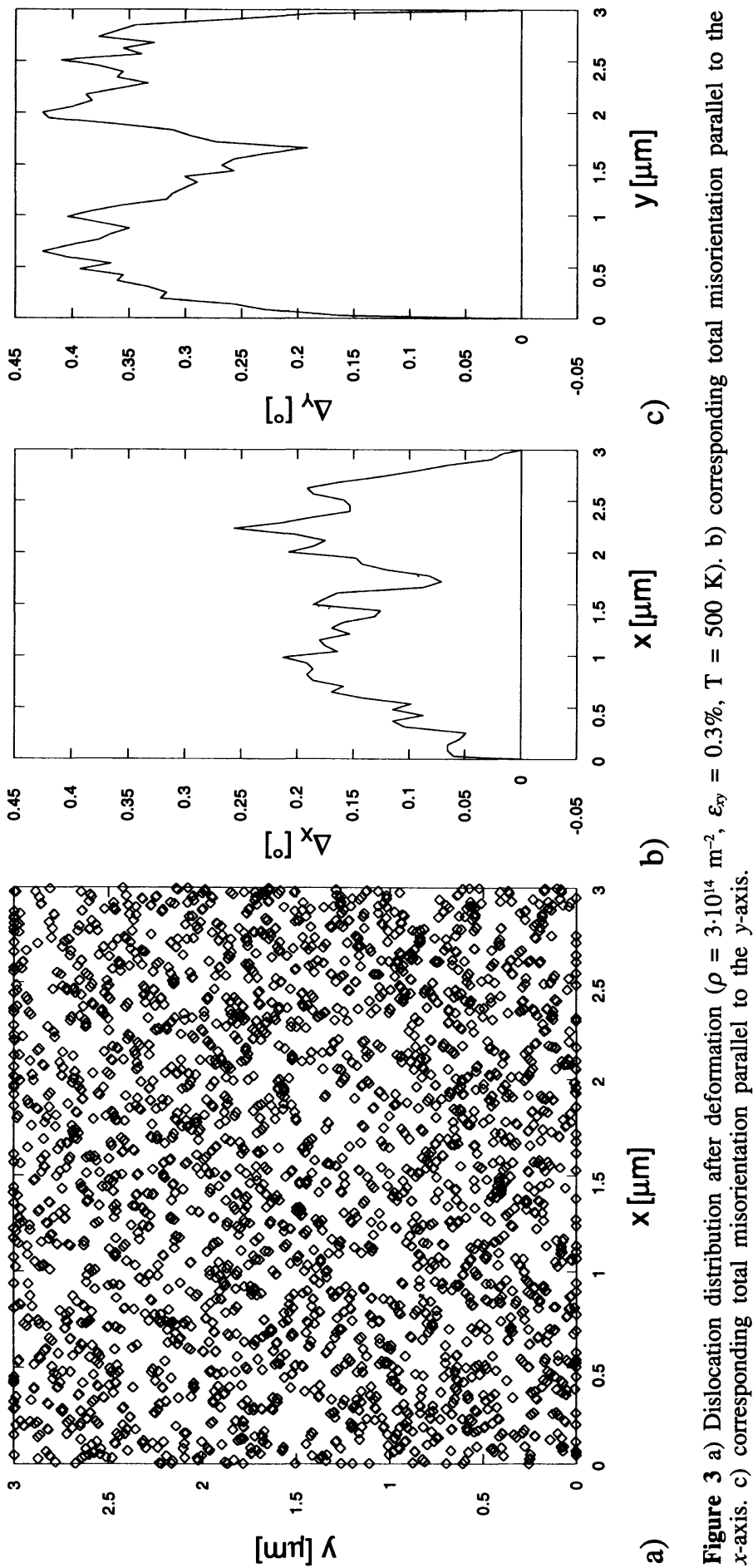

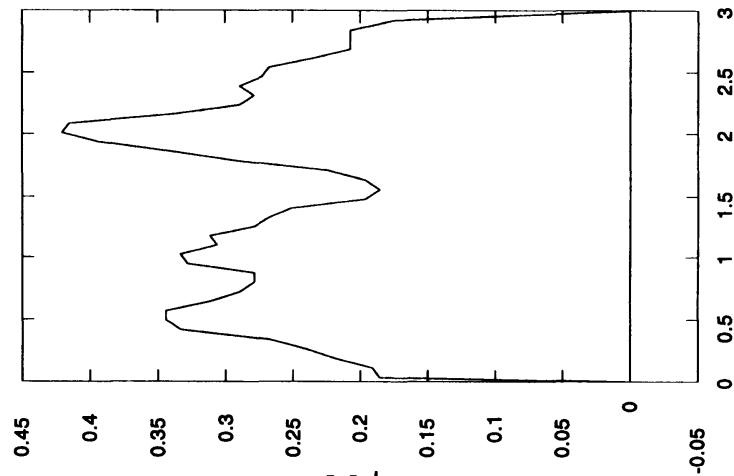

[0] ${ }^{\wedge} \nabla$
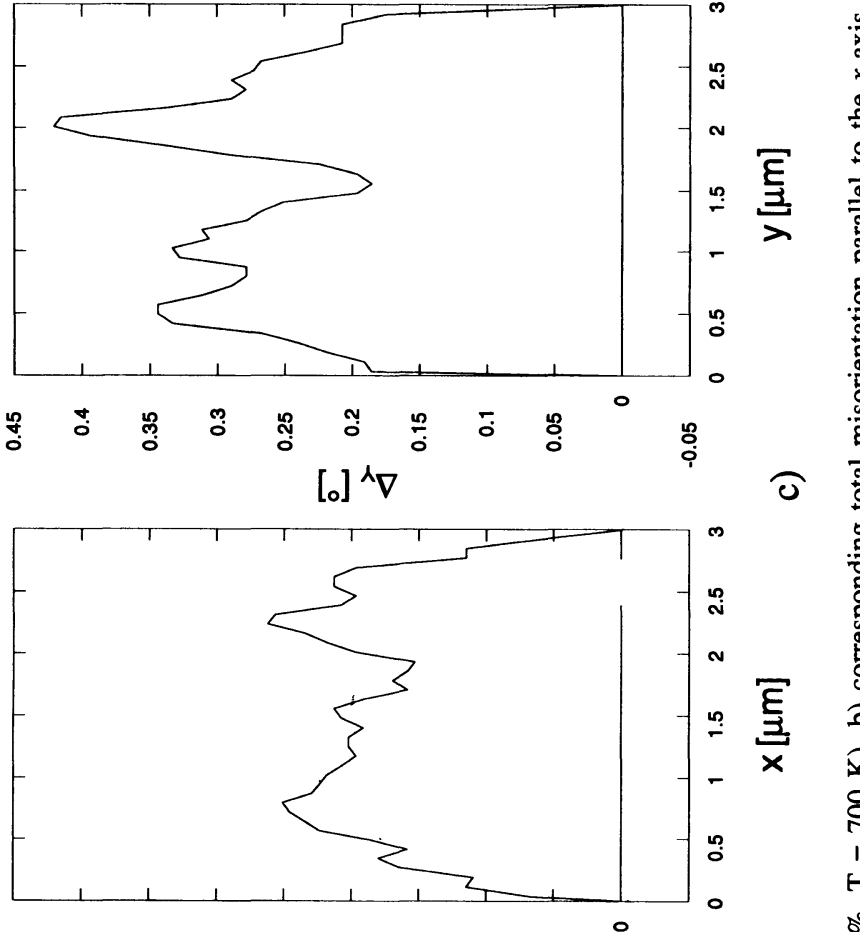

㿝

[0] ${ }^{\mathrm{X}} \nabla$

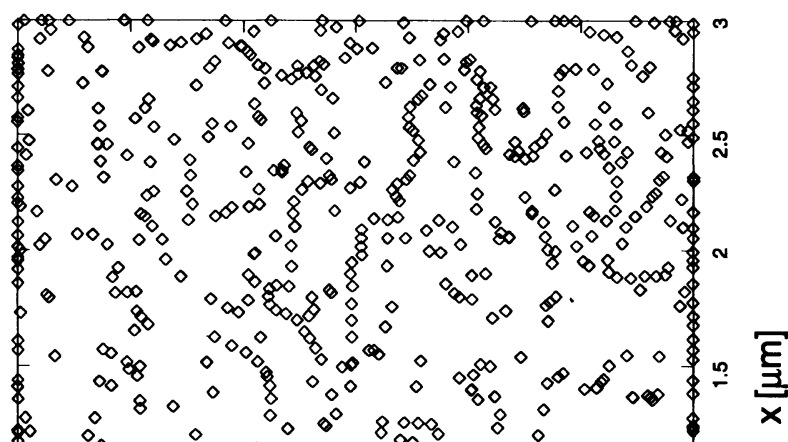


During the subsequent annealing treatment the dislocation density is reduced by about $45 \%\left(\rho=1.7 \cdot 10^{14} \mathrm{~m}^{-2}\right.$, Figure $\left.4 \mathrm{a}\right)$ while the misorientation distribution is only slightly affected $\left(\Delta_{\max } \approx 0.42^{\circ}\right.$, Figures $4 \mathrm{~b}$ and $\left.4 \mathrm{c}\right)$. This weak change in the local misorientations during recovery is even more evident from figure 5 where the misorientations along the $y$-axis are plotted into one diagram for all three states of the experiment. The maximum difference found in the misorientation curves of the last two states amounts to $0.1^{\circ}$. An analysis of the course of the misorientation plot shows that the misorientation is constant over most of the grain but that there are large orientation gradients in the direct vicinity of the large angle grain boundaries, i.e. at the walls of the simulation cell. This becomes even more evident in figure 6 where the misorientation increments, $\delta_{y}$, are plotted for every cell. The maximum value of $\delta_{y}$ inside the grain amounts to $0.8^{\circ}$ (the average value is even smaller) while at the grain boundary values of as much as $1.9^{\circ}$ are found. These results are in reasonable agreement with experimental findings (Boeslau and Raabe 1994, Rosen et al. 1995).

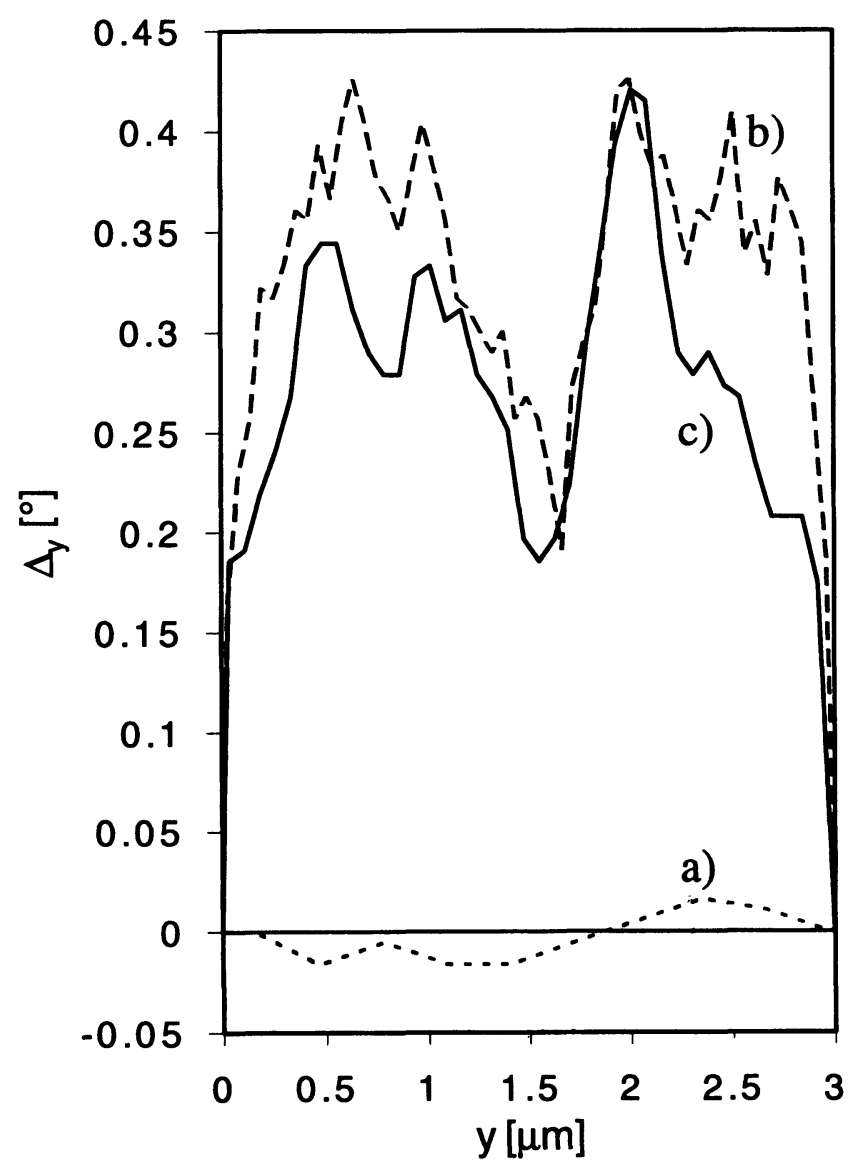

Figure 5 Misorientation along the $y$-axis. a) initial distribution b) after deformation c) after anneal. 


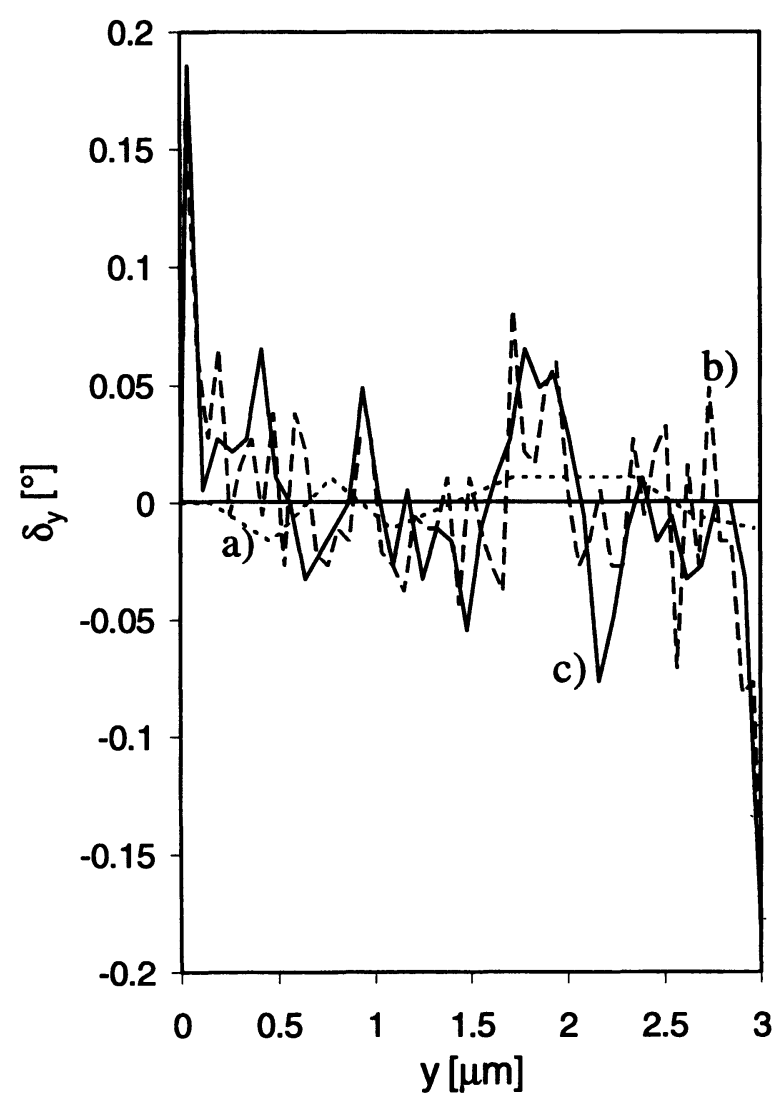

Figure 6 Misorientation increments along the $y$-axis. a) initial distribution b) after deformation c) after anneal.

\section{DISCUSSION}

It was shown that microtexture prediction by means of 2 dimensional dislocation dynamics simulation reveals reasonable results even with a considerably simplified choice of geometry for the subdivision of the simulated grain. The simulation is capable of showing how misorientations build up during deformation and how microtexture is influenced by static recovery. The influence of the grain orientation can be studied by choosing different sets of active glide systems.

Nevertheless there still are multiple opportunities to further increase the quality of the simulation results. The simulation of the dislocation distribution evolution can be improved especially with regard to the implementation of a more realistic model for the multiplication process. Furthermore, the boundary conditions used should additionally consider the influence of the neighboring grains. Therefore the shape of the simulation cell should not be preserved during the entire simulation but should for example allow for deformation. The grain rotation could be also taken into account. 
The evaluation of the misorientation from the dislocation distribution can be easily improved by the choice of the geometry of the cells used to divide the grain. For example one could choose squares instead of elongated rectangulars. This would make it possible to calculate the misorientations as a function of both $x$ and $y$ at the same time.

\section{Acknowledgments}

The authors gratefully acknowledge the support by the Deutsche Forschungsgemeinschaft (DFG) through the Collaborative Research Center 370 "Integrative Werkstoffmodellierung". The authors are grateful to Prof. G. Gottstein, Prof. D. Srolovitz and Prof. H. Mecking for helpful discussions.

\section{References}

Ashby, M. F. (1970). The Deformation of Plastically Non-Homogeneous Materials. Phil. Mag., 21 pp. 399-424.

Beaudoin, Mecking and Kocks (1995) to be published.

Boeslau, J. and Raabe, D. (1994). Development of Microtextures in Cold Rolled Iron Oligocrystals. Material Science Forum, 157-162 pp. 157-162.

Bunge, H. J. (1965). Zur Darstellung allgemeiner Texturen. Zeitschrift für Metallkunde, 56 pp. 872-874.

Bunge, H. J. (1982). Texture Analysis in Materials Science, Butterworths, London.

Dingley, D. J. (1984). Diffraction from Sub-Micron Areas using Electron Backscattering in a Scanning Electron Microscope. Scanning Electr. Microsc. /II pp. 569-575.

Dingley, D. J. and Randle, V. (1992). Microtexture Determination by Electron Back-Scatter Diffraction. Journal of Materials Science, 27 pp. 4545-4566.

Engler, O. and Gottstein, G. (1992). A new Approach in Texture Research: Local Orientation Determination with EBSP. Steel Research, 63 pp. 413-418.

Essmann, U. and Mughrabi, H. (1979). Annihilation of Dislocation During Tensile and Cyclic Deformation and Limits of Dislocation Densities. Phil. Mag. A 40 pp. 731-756.

Hirth, J. P. and Lothe, J. (1968). Theory of Dislocations, McGraw-Hill Book Company.

Hjelen, J., Orsund, R. and Nes, E. (1991). On the Origin of Recristallisation Textures in Aluminum. Acta Metall., 39 pp. 1377-1404.

Humphreys, F. J. (1984). A Method for Determining Textures by Electron Diffraction. Proc. ICOTOM 7 pp. 771-776.

Juul Jensen, D. (1992). Modelling of Microstructure Development During Recristallisation. Scripta Metall., 27 pp. 1551-1556.

Köhler, U. and Bunge, H. J. (1994). Calculation of the Recristallisation Textures of Cubic Metals. Material Science Forum, 157-162 pp. 1803-1808.

Lee, C. S., Duggan, D. J. and Smallman. R. E (1993). A Theory of Deformation Banding in Cold Rolling Acta Metall., 41 pp. 2265-2270.

Marx, V., Raabe, D. and Gottstein, G. (1995). Simulation of the Influence of Recovery on the Texture Development in Cold Rolled BCC Alloys During Annealing. Proceedings 16th RIS $\emptyset$ Int. Sympos. on Mat. Science: Microstructural and Crystallographic Aspects of Recristallisation, RIS $\emptyset$ Nat. Lab., Roskilde, Denmark pp. 461-466.

Mughrabi, H. (1983). Dislocation Wall and Cell Structures and Long-Range Internal Stresses in Deformed Metal Crystals. Acta Metall., 31 pp. 1367-1379.

Nabarro, F. R. N. (1952). The Mathematical Theory of Stationary Dislocations. Adv. Phys., 1 pp. 269-378.

Nye. J. F. (1953). Some Geometrical Relations in Dislocated Crystals. Acta Metall., 1 pp. $153-162$.

Raabe, D. (1994). Simulation and Experimental Examination of the Evolution of Orientation Gradients in Single Grains During Rolling of BCC Polycristals. physica status solidi (b) 181 pp. 291-299.

Raabe D. (1996). to be published.

Rosen, G. I., Juul Jensen, D., Hughes, D. A. and Hansen, N. (1995). Microstructure and Local Crystallography of Cold Rolled Aluminum. Acta Metall., 43 pp. 2563-2579.

Schwarzer R. (1991). A Review of the Analysis of Local Texture by Electron Diffraction. Texture and Microstr., 14-18 pp. 85-90.

Taylor G. I. (1938). Plastic Strain in Metals. J. Inst. Met., 62 pp. 307-312. 
Venables, J. and Harland, C. (1973). Electron Back-Scattering Patterns - A new Technique for Obtaining Crystallographic Information in the Scanning Electron Microscope Phil. Mag., 27 pp. 1193-1200. Zehetbauer, M. and Seumer, V. (1993). Cold Work Hardening in Stages IV and V of F.C.C. Metals - I. Experiments and Interpretation. Acta Metall., 41 pp. 577-588.

Zehetbauer, M. (1993). Cold Work Hardening in Stages IV and V of F.C.C. Metals - II. Model Fits and Physical Results. Acta Metall., 41 pp 589-599. 